\title{
Systems Reconsolidation Reveals a Selective Role for the Anterior Cingulate Cortex in Generalized Contextual Fear Memory Expression
}

\author{
Einar Ö Einarsson ${ }^{*}, 1,2$, Jennifer Pors' and Karim Nader,, \\ 'Department of Psychology, McGill University, Montreal, QC, Canada
}

\begin{abstract}
After acquisition, hippocampus-dependent memories undergo a systems consolidation process, during which they become independent of the hippocampus and dependent on the anterior cingulate cortex (ACC) for memory expression. However, consolidated remote memories can become transiently hippocampus-dependent again following memory reactivation. How this systems reconsolidation affects the role of the ACC in remote memory expression is not known. Using contextual fear conditioning, we show that the expression of 30-day-old remote memory can transiently be supported by either the ACC or the dorsal hippocampus following memory reactivation, and that the ACC specifically mediates expression of remote generalized contextual fear memory. We found that suppression of neural activity in the ACC with the AMPA/kainate receptor antagonist 6-cyano-7-nitroquinoxaline-2,3-dione (CNQX) impaired the expression of remote, but not recent, contextual fear memory. Fear expression was not affected by this treatment if preceded by memory reactivation $6 \mathrm{~h}$ earlier, nor was it affected by suppression of neural activity in the dorsal hippocampus with the GABA-receptor agonist muscimol. However, simultaneous targeting of both the ACC and the dorsal hippocampus $6 \mathrm{~h}$ after memory reactivation disrupted contextual fear memory expression. Second, we observed that expression of a 30-day-old generalized contextual fear memory in a novel context was not affected by memory reactivation $6 \mathrm{~h}$ earlier. However, intra-ACC CNQX infusion before testing impaired contextual fear expression in the novel context, but not the original training context. Together, these data suggest that although the dorsal hippocampus may be recruited during systems reconsolidation, the ACC remains necessary for the expression of generalized contextual fear memory.
\end{abstract}

Neuropsychopharmacology (2015) 40,480-487; doi: I0.1038/npp.2014.197; published online 3 September 2014

\section{INTRODUCTION}

Lesions or pharmacological inactivation of the hippocampus preferentially impact recently acquired contextual fear memory (Anagnostaras et al, 1999; de Oliveira Alvares et al, 2012; Goshen et al, 2011; Kitamura et al, 2009; Ward et al, 1999; Wiltgen et al, 2010; Winocur et al, 2009). Conversely, transient disruption of the anterior cingulate cortex (ACC) impairs the expression of remote, but not recent memory (Frankland et al, 2004; Goshen et al, 2011). Such findings have led to the idea that the hippocampus serves a critical role in integrating information from a distributed cortical network representing various features of a memory, and that over time, the medial prefrontal cortex (including the ACC) gradually takes over this integrative role, allowing

*Correspondence: Dr E Ö Einarsson, INSERM U862, Neurocentre Magendie, I 46 Rue Léo Saignat, Bordeaux cedex 33077, France, Tel: +33557 573719, Fax: +33557573669,

E-mail: einar.einarsson@inserm.fr or Dr $\mathrm{K}$ Nader, Department of Psychology, McGill University, 1205 Dr. Penfield Avenue, Montreal, QC, Canada H3A IBI. E-mail: karim.nader@mcgill.ca

${ }^{2}$ Current address: INSERM U862, Neurocentre Magendie, I46 Rue Léo Saignat, Bordeaux cedex 33077, France

Received 30 March 20 I4; revised 28 July 20 I4; accepted 3 I July 20 I4; accepted article preview online 5 August 2014 the memory to function independently of the hippocampus (Frankland and Bontempi, 2005). In support of this view, imaging studies have revealed sequential activity in area CA1 in the hippocampus and ACC for recent and remote time points after learning, respectively, in terms of immediate-early gene expression (Frankland et al, 2004), and dendritic spine growth (Vetere et al, 2011). This transition has been suggested to reflect systems consolidation, during which post-training hippocampal replay of episodic or detailed memory leads to memory trace reactivation in the cortex, promoting the development of a more schematic cortical representation (McClelland et al, 1995; Winocur et al, 2010).

Recent studies suggest that retrieval can make remote hippocampus-independent contextual fear memory again dependent on the hippocampus for a brief time (Debiec et al, 2002; Winocur et al, 2009). Such renewed dependence on the hippocampus after memory reactivation is called systems reconsolidation. Specifically, these studies showed that electrolytic lesions of the dorsal hippocampus impaired remote memory only when applied after memory reactivation. It remains unclear, however, whether remote memory reactivation also affects the role of the ACC. For example, it is not known whether the ACC critically supports the remote memory expression after reactivation, or if the 
reactivated remote memory only requires the hippocampus, as is the case for recent memory.

To examine how remote memory reactivation affects the brain structures supporting contextual fear memory expression, we tested whether pharmacological suppression of neural activity in the ACC and/or dorsal hippocampus disrupts expression of remote contextual fear memory following memory reactivation. First, we found that after remote memory reactivation, suppression of neural activity in the ACC impaired memory expression when tested $24 \mathrm{~h}$, but not $6 \mathrm{~h}$, later. Second, we found that only simultaneous suppression of neural activity in the ACC and the dorsal hippocampus impaired memory expression $6 \mathrm{~h}$ after memory reactivation, suggesting that memory expression can be supported by either structure alone. Third, we found that pre-test suppression of neural activity in the ACC $6 \mathrm{~h}$ after memory reactivation impaired fear expression in a novel context, but did not affect memory expression in the training context, suggesting that the ACC specifically mediates generalized fear memory expression.

\section{MATERIALS AND METHODS}

\section{General Behavioral Procedures}

Subjects. Male Sprague-Dawley rats (Charles River, SaintConstant, PQ) weighing at least $250 \mathrm{~g}$ at the start of training were used in experiments. Animals were housed individually and maintained on a 12/12 h light/dark cycle (lights on at 0700 hours) with food and water provided ad libitum. The rats were handled daily on 5 consecutive days before training for $\sim 2 \mathrm{~min}$. All procedures were in accordance with the Canadian Council on Animal Care Guide, and were approved by the McGill University Animal Care and Use Committee.

Surgery and histology. Under ketamine $(55 \mathrm{mg} / \mathrm{kg})$, xylazine $(3.33 \mathrm{mg} / \mathrm{kg})$, and domitor $(27 \mathrm{mg} / \mathrm{kg})$ anesthesia, 26-G stainless steel cannulae were implanted bilaterally into the ACC (injector coordinates: AP $2.6 \mathrm{~mm}$ relative to bregma; $\mathrm{ML} \pm 0.7 \mathrm{~mm} ; \mathrm{DV}-1.6 \mathrm{~mm}$ to dura), the dorsal hippocampus ( $\mathrm{AP}-3.6 \mathrm{~mm}$ relative to bregma; $\mathrm{ML}$ $\pm 3.1 \mathrm{~mm}$; DV $-3.1 \mathrm{~mm}$ to dura, and $-1.6 \mathrm{~mm}$ to dura for the control group receiving infusions dorsally to the dorsal hippocampus). Rats were given a week to recover. In experiments testing recent memory (3 days postconditioning), rats were operated on before conditioning. In experiments testing remote memory, surgery was performed between conditioning and testing. Guide cannulae were fixed to the skull with dental cement and stainless steel screws. At the end of the experiment, animals were transcardially perfused with physiological saline followed by $10 \%$ formal-saline. Brains were sectioned at $50 \mu \mathrm{m}$ thickness and stained with formol-thionin and examined by light microscopy for verification of cannula placement.

Drugs and infusions. In a mixed design of two test sessions, rats received infusions of either drug or vehicle into the ACC before test 1 , and then the opposite treatment before test 2 . Neural activity was suppressed using the AMPA/kainate receptor antagonist 6-cyano-7-nitroquinoxaline-2,3-dione (CNQX), a drug that has been shown to metabolize rapidly within a few hours after cortical infusions (Attwell et al, 1999; Honore et al, 1988). Other drugs, such as muscimol, are known to suppress neuronal activity for longer periods. In one experiment involving the dorsal hippocampus, we used muscimol for suppressing neuronal activity during test 2 as the drug's effects and diffusion have been extensively characterized for that structure. A previous study (Corcoran et al, 2005) targeting the dorsal hippocampus, using the same dose as in this study with autoradiographic analysis of the spread of $[3 \mathrm{H}]$ muscimol, found that the drug did not bind to other subcortical structures, although it did diffuse to more dorsal cortical regions. To further test the functional effects of possible dorsal diffusion from infusion to the dorsal hippocampus, we included a group receiving muscimol infusion into cortical regions dorsal to the dorsal hippocampus.

Drugs were infused via an infusion pump at a rate of $0.25 \mu \mathrm{l} / \mathrm{min}$. CNQX disodium salt (Tocris, Ellisville, MO) was dissolved in nanopure water and infused at $2.5 \mu \mathrm{g} /$ $0.25 \mu \mathrm{l}$ per side. Muscimol was dissolved in artificial cerebrospinal fluid and injected at $0.5 \mu \mathrm{g} / 0.5 \mu \mathrm{l}$ per side. Representative photomicrographs of infusion sites marked with methylene-blue solution in the ACC or the dorsal hippocampus are depicted in Supplementary Figure 1.

Apparatus. The training context consisted of rodent conditioning cages with clear Plexiglas walls and a metal grid floor (Coulbourn Instruments) that was enclosed within a sound-attenuating chamber in a well-lit room. The cages were dimly lit with a single house light, had a fan on for background noise, and were scented with diluted vanilla. The novel context was in a dark room and consisted of a rodent conditioning cage (Med Associates, St Albans, VT) with a striped Plexiglas wall front, aluminum sides, clear Plexiglas top, curved gray plastic back, and plastic floor covered with bedding. The cage was enclosed in a soundattenuating chamber with blinking white LED light above the cage.

Behavioral procedures. The day before conditioning, rats were habituated to the conditioning chamber (training context) for $5 \mathrm{~min}$. For conditioning, rats received eight unsignaled foot-shocks $(1.5 \mathrm{~mA}, 1 \mathrm{~s})$ at $62 \mathrm{~s}$ intervals $2 \mathrm{~min}$ after being placed in the context and then remained there for additional $30 \mathrm{~s}$. For all tests, freezing (defined as the complete absence of movement, except that of respiration) was scored with an instantaneous time-sampling procedure, in which each animal was observed as either freezing or not every $5 \mathrm{~s}$. All test and memory reactivation sessions were 3 min long.

Experimental procedures. In the first experiment, we examined the effects of suppressing neural activity in the ACC on fear memory expression during tests of recent and remote memory, and during a test following remote memory reactivation. For test of recent 3-day-old memory, rats received intra-ACC infusions of $\mathrm{CNQX}(n=10)$ or its vehicle $(n=10)$ before testing. To test ACC involvement in 30 -day-old remote memory expression and the effects of remote memory reactivation on expression $6 \mathrm{~h}$ later, separate groups received intra-ACC infusion of CNQX or 
its vehicle before testing, and then the opposite treatment $6 \mathrm{~h}$ later (first infusion/second infusion: $\mathrm{CNQX/vehicle:}$ $n=6$; vehicle/CNQX: $n=7$ ). For test of ACC involvement in remote memory expression and expression $24 \mathrm{~h}$ following remote memory reactivation, separate groups received intra-ACC infusions of CNQX or its vehicle before testing, and then the opposite treatment $24 \mathrm{~h}$ later (CNQX/vehicle: $n=11$; vehicle/CNQX: $n=13$ ).

In the second experiment, we examined the effects of suppressing neural activity in the ACC and/or dorsal hippocampus on fear memory expression following memory reactivation. Thirty days after conditioning, we placed the rats in the training context for a memory reactivation trial (test 1). Six hours later, we infused either drug or its vehicle simultaneously into the ACC and the dorsal hippocampus before test 2, dividing the animals in five groups (ACC infusion/dorsal hippocampus infusion, respectively): CNQX/ muscimol $(n=6)$, vehicle/muscimol $(n=6), \mathrm{CNQX} /$ vehicle $(n=7)$, vehicle/vehicle $(n=6)$, in addition to a control group where infusions were targeted dorsal to the dorsal hippocampus; CNQX/dorsal-control muscimol $(n=6)$.
In the third experiment, we examined the effects of suppressing neural activity in the ACC on generalized contextual fear memory expression following remote memory reactivation. To first evaluate changes in context generalization over time, rats were tested in either the training context or a novel context at either 3 days (training CXT, $n=8$; novel CXT, $n=8$ ) or 30 days (training CXT, $n=11$; novel CXT, $n=11$ ) following contextual fear conditioning. To test the effects of memory reactivation on context generalization, rats were returned to the training context 30 days after training for a memory reactivation trial (test 1). Separate groups were then tested in either the training context or the novel context $6 \mathrm{~h}$ later (test 2; training CXT, $n=11$; novel CXT, $n=11$ ). To test the effects of blocking AMPA/kainate-receptor-mediated neural activity in the ACC on generalized contextual fear expression $6 \mathrm{~h}$ following memory reactivation, four groups of rats were then tested in either the training context or the novel context after receiving local ACC infusion of CNQX (training CXT, $n=6$; novel CXT, $n=7$ ) or its vehicle (training CXT, $n=7$; novel CXT, $n=7$ ). a

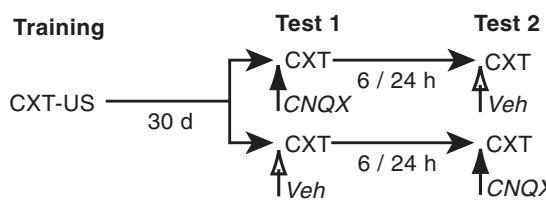

b

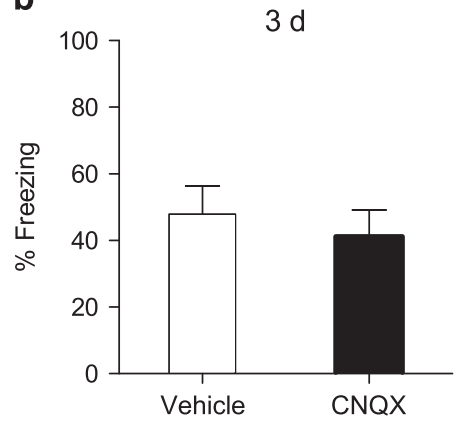

d

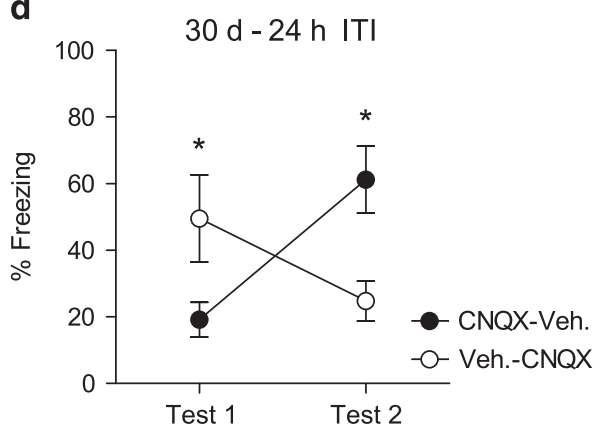

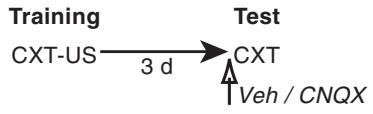

C

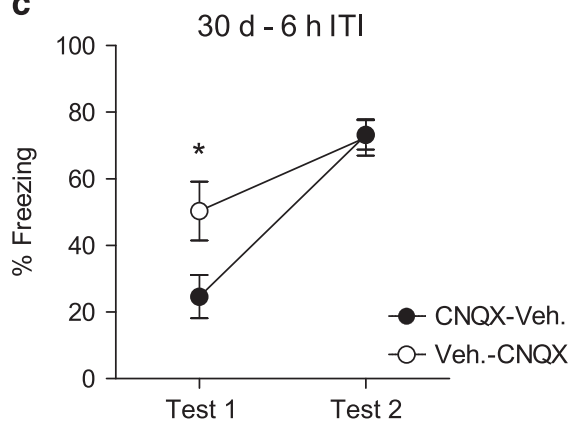

e

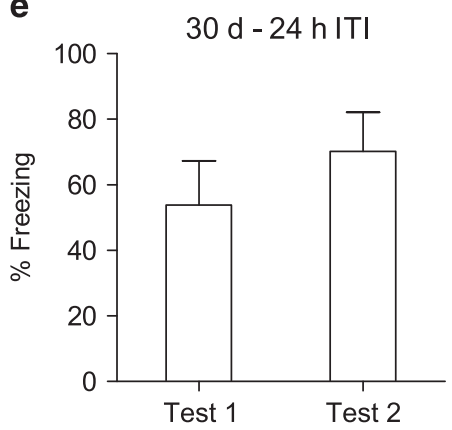

Figure I Effects of CNQX infusion into the ACC on recent and remote contextual fear memory expression, and expression following remote memory reactivation. (a) Experimental designs used in testing 30-day-old recent memory (left) and 3-day-old remote memory (right). (b) At 3 days after training, pretest CNQX infusions into the ACC did not impair contextual fear memory expression. (c) At 30 days after training, pre-test CNQX infusions into the ACC impaired contextual fear memory expression (test I), but if preceded by memory reactivation $6 \mathrm{~h}$ earlier (vehicle group on test I; $6 \mathrm{~h}$ inter-test-interval, ITI), the same treatment did not impair contextual fear memory expression (test 2). (d) At 30 days after training, pre-test CNQX infusions into the ACC impaired contextual fear memory expression (test I), and when preceded by memory reactivation $24 \mathrm{~h}$ earlier (vehicle group on test I), the same treatment was similarly effective in impairing contextual fear memory expression. (e) Testing 30-day-old contextual fear memory twice with $24 \mathrm{~h}$ inter-test-interval did not impair contextual fear memory expression on the second test. $* P<0.05$. Data are means \pm SEM. 
Statistical analysis. Paired and independent group's $t$-tests were performed (two-tailed), in addition to one-way independent groups and two-way mixed-factor ANOVAs. Significant interactions were followed by Tukey's post-hoc test where appropriate.

\section{RESULTS}

AMPA/Kainate-Receptor Blockade in the ACC Disrupts Remote Fear Memory Expression Preceded by Memory Reactivation $24 \mathrm{~h}$, but not $6 \mathrm{~h}$, Earlier

To directly test whether the ACC remains critical for memory expression following remote memory reactivation, we suppressed AMPA/kainate-receptor-mediated neural activity in the ACC using the reversible antagonist CNQX (Figure 1a). In addition to systems reconsolidation, during which memory expression becomes again transiently dependent on the hippocampus, memory reactivation also leads to cellular reconsolidation, which requires less than $6 \mathrm{~h}$ to stabilize reactivated memory (Flavell et al, 2011; Monfils et al, 2009; Nader et al, 2000). In order to avoid overlapping with an active cellular reconsolidation process during our test, in which we aimed to assess the effects of systems reconsolidation, we tested the effects of remote memory reactivation on subsequent memory expression $6 \mathrm{~h}$ later. Rats were tested 30 days after contextual fear conditioning (test 1 ) and again at $6 \mathrm{~h}$, or $24 \mathrm{~h}$, later (test 2). One group received CNQX infusions before test 1 and vehicle before test 2 (group CNQX-vehicle), whereas another group received vehicle infusions before test 1 and CNQX before test 2 (group vehicle-CNQX). Thus, test 1 served both as a test of ACC involvement in remote memory retrieval (between-group comparison of CNQX and vehicle infusion on test 1), and as a memory reactivation trial for the group receiving vehicle on test 1 before later receiving CNQX before test 2, allowing us to test the effect of memory reactivation (test 1) on ACC involvement in later memory retrieval (test 2).

We also tested the involvement of the ACC in recent memory expression and found that pre-test CNQX infusion into the ACC did not impair memory expression $(P>0.05$; Figure $1 \mathrm{~b})$. At 30 days, with a 6-h inter-test interval (Figure 1c), $2 \times 2$ mixed design ANOVA with test (test $1 v \mathrm{~s}$ test 2) as a within-subjects factor and treatment group (group CNQX-vehicle $v s$ group vehicle-CNQX) as a between-subjects factor, revealed a significant group by test interaction $\left(\mathrm{F}_{1,22}=4.9 ; P<0.05\right)$, with post-hoc analyses indicating that CNQX infusion into the ACC impaired remote memory expression (test 1 , vehicle vs CNQX; $P<0.05$ ), whereas ACC CNQX infusion $6 \mathrm{~h}$ later (test 2 for group receiving vehicle on test 1 ), did not disrupt memory expression (test 2; vehicle $v s$ CNQX; $P<0.05$ ).

When the inter-test interval of remote memory was increased to $24 \mathrm{~h}$ using a $2 \times 2$ mixed design as before (Figure 1d), ANOVA revealed a significant group by test interaction $\left(\mathrm{F}_{1,11}=19.5 ; P<0.05\right)$, with post-hoc analyses indicating that $\mathrm{CNQX}$ infusion into the ACC impaired remote memory retrieval on test 1 (vehicle vs CNQX; $P<0.05$ ), and that pre-test CNQX infusion $24 \mathrm{~h}$ following memory reactivation (test 1 vehicle group) now impaired memory expression (test 2 , vehicle vs CNQX; $P<0.05$ ).
Impaired memory expression $24 \mathrm{~h}$ after memory reactivation was not due to delayed expression of fear extinction, as a separate experiment showed that pre-test vehicle infusion into the ACC at 30 days and again $24 \mathrm{~h}$ later did not impair fear memory expression on test $2(P>0.05$; Figure $1 \mathrm{e})$. These results suggest that following reactivation of 30-dayold remote memory, memory can be transiently expressed without the ACC $6 \mathrm{~h}$ later, before returning to an ACCdependent state after $24 \mathrm{~h}$. Similar results were observed 6 and $24 \mathrm{~h}$ following the reactivation of a 45 -day-old remote memory (Supplementary Figure 2).

\section{Remote Fear Memory Expression 6 h Following Memory Reactivation can be Supported by either the ACC or the Dorsal Hippocampus}

We next tested if the dorsal hippocampus mediates memory expression during suppression of neural activity in the ACC $6 \mathrm{~h}$ after memory reactivation of a 30 -day-old memory by suppressing neural activity in either structure, or both. Six hours after memory reactivation (ACC vehicle treatment as in the previous experiment), different groups were infused with CNQX into the ACC and/or muscimol into the dorsal hippocampus, or their vehicle, before test 2 (Figure 2a). To control for anatomical specificity of the dorsal hippocampal infusion, we included a fifth group that was infused with muscimol immediately dorsal to the dorsal hippocampus, in addition to CNQX infusion into the ACC. We found that only simultaneous CNQX and muscimol infusion into the ACC and the dorsal hippocampus, respectively, disrupted remote memory expression $6 \mathrm{~h}$ after memory reactivation

a

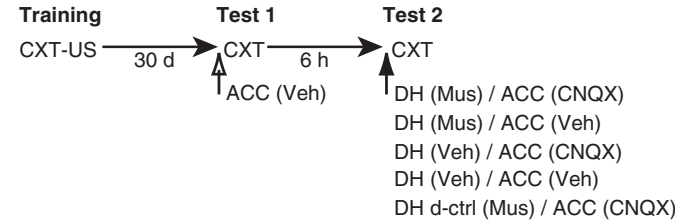

b

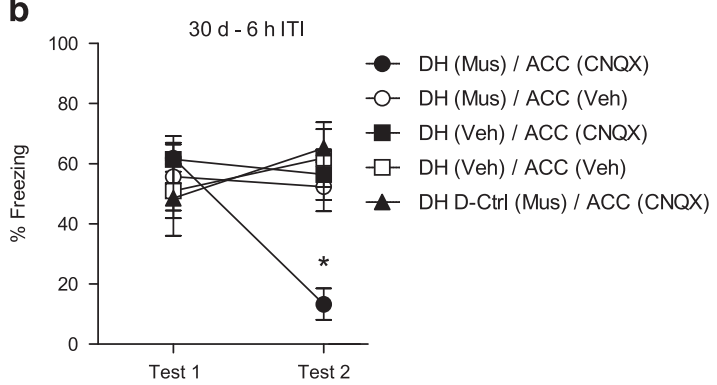

Figure 2 Effects of CNQX infusion into the ACC and muscimol infusion into the dorsal hippocampus on remote contextual fear memory preceded by memory reactivation. (a) Experimental design used with data presented below. (b) At 30 days after training and preceded by memory reactivation $6 \mathrm{~h}$ earlier, only simultaneous pre-test infusions of CNQX into the ACC and muscimol into the dorsal hippocampus impaired contextual fear memory expression, whereas simultaneous infusions of either drug alone with the vehicle of the other drug did not impair contextual fear memory expression. A control for anatomical specificity of dorsal hippocampus infusions, where muscimol was infused dorsal to the dorsal hippocampus with CNQX infused into the ACC, did not impair contextual fear memory expression. $* P<0.05$. Data are means \pm SEM. 
(group $\times$ test interaction, $\mathrm{F}_{4}, 28=6.8 ; P<0.01$; post hoc, $P<0.05)$, indicating that $6 \mathrm{~h}$ following memory reactivation, contextual fear memory expression in the training context can be supported without either the ACC or the dorsal hippocampus, but not without both structures (Figure 2b). These findings are consistent with the idea that the hippocampus and ACC have a critical integrative role in a distributed cortical network, each supporting recent and remote memory, respectively (Frankland and Bontempi, 2005), and furthermore suggest that $6 \mathrm{~h}$ following remote memory reactivation, both structures can serve this integrative role independently of each other.

\section{AMPA/Kainate-Receptor Blockade in the ACC $6 \mathrm{~h}$ Following Remote Memory Reactivation Selectively Disrupts Generalized Contextual Fear}

Contextual memories are known to become less precise in the weeks following learning in rodents (Biedenkapp and Rudy, 2007; Riccio et al, 1992; Ruediger et al, 2011; Wiltgen et al, 2010; Winocur et al, 2009; Winocur et al, 2007), such that animals increasingly generalize the conditioned response to a novel context similar to the training context, and eventually show comparable conditioned responding to both contexts. In light of our findings that suppressing neural activity in the ACC by blocking AMPA/kainate receptors was ineffective in disrupting contextual fear expression in the original training context $6 \mathrm{~h}$ following remote memory reactivation, we next tested if AMPA/ kainate-receptor blockade in the ACC at this time point would affect generalized contextual fear memory expression. To this end, we first characterized contextual discrimination at a recent ( 3 days) and remote time point (30 days) after conditioning (Figure 3a). Using an independentgroups ANOVA design, we found that although freezing in the training context remained high at 3 and 30 days after conditioning, freezing in a novel context increased from lower levels of expression at 3 days to similarly elevated fear expression in the training context at 30 days (context $\times$ test day interaction, $\mathrm{F}_{1,34}=7.4 ; P<0.01$; post-hoc, $\left.P<0.01\right)$.

Next, we examined the effect of remote memory reactivation on contextual generalization $6 \mathrm{~h}$ later (Figure $3 \mathrm{~b}$ ). At 30 days after contextual fear conditioning, we exposed two groups of rats to the original training context for memory reactivation, and then $6 \mathrm{~h}$ later, one group was tested again in the training context, whereas the other group was tested in a novel context. A mixed design ANOVA, with test as the between-groups factor and context as the within-groups factor, revealed a significant main effect of test $\left(\mathrm{F}_{1,20}=17.2\right.$; $P<0.001$ ) but no difference in fear responding to the training and novel context preceded by memory reactivation 6 h earlier (context $\times$ test interaction $P>0.05$ ), suggesting that remote memory reactivation does not affect generalized contextual fear expression $6 \mathrm{~h}$ later.

Finally, we tested the effect of ACC AMPA/kainatereceptor blockade with CNQX on generalized contextual fear expression $6 \mathrm{~h}$ after remote memory reactivation (Figures $3 c$ and d). A mixed design ANOVA revealed that $6 \mathrm{~h}$ after memory reactivation in the original training context, CNQX infusion into the ACC disrupted fear expression in the novel context, but not in the training context (context $\times$ test interaction $\mathrm{F}_{1,11}=10.33 ; P<0.01$; post hoc, $P<0.01)$. Vehicle infusions into the ACC $6 \mathrm{~h}$ after memory reactivation had no effect on subsequent responding to the training or novel context (test main effect $P>0.05$; context $\times$ test interaction $P>0.05$ ). This suggests that, following remote memory reactivation, AMPA/kainate-receptor blockade in the ACC selectively impairs fear memory expression in a novel context but spares memory expression in the original training context, which is then supported by the dorsal hippocampus during suppression of neural activity in the ACC (Figure 2b).

\section{DISCUSSION}

The present study examined how memory reactivation affects the role of the ACC in the expression of contextual fear memory. We found that suppressing neural activity in the ACC disrupted the expression of remote contextual fear memory. However, if preceded by memory reactivation, suppressing neural activity in the ACC $6 \mathrm{~h}$ later did not impair remote memory expression, whereas at $24 \mathrm{~h}$ the same treatment became again effective in disrupting memory expression. Only by simultaneously disrupting neural activity in the dorsal hippocampus and the ACC $6 \mathrm{~h}$ after memory reactivation was memory expression disrupted, whereas targeting the dorsal hippocampus alone was ineffective.

Our observation of increased involvement of the ACC in remote memory is in line with previous research showing that, over time (after 2 weeks in rodents), the ACC becomes more involved in supporting contextual fear memory (Frankland et al, 2004; Goshen et al, 2011), whereas at the same time the involvement of the hippocampus is reduced (Anagnostaras et al, 1999; de Oliveira Alvares et al, 2012; Goshen et al, 2011; Kitamura et al, 2009; Ward et al, 1999; Wiltgen et al, 2010; Winocur et al, 2009). Our findings are also consistent with the idea that the hippocampus is critical for integrating information in a distributed cortical network representing various features of a memory, but as the memory trace matures, the ACC gradually takes over this integrative role and becomes critical for the expression of remote memory (Frankland and Bontempi, 2005). However, our findings also show that expression of remote contextual fear memory can be supported by either the ACC or the dorsal hippocampus $6 \mathrm{~h}$ following memory reactivation; therefore, our results support the view that remote memory reactivation recruits the hippocampus again, albeit transiently (Debiec et al, 2002; Winocur et al, 2009). It is possible that in order to update existing memory, the hippocampus becomes briefly involved in incorporating new information into a hippocampal-cortical memory trace, after which the hippocampus, although it continues to contribute to the memory trace itself, is no longer necessary for its expression (Hardt et al, 2010). Findings of rapid systems-consolidation in a hippocampus-dependent flavor-place-pairing task support this model. In this paradigm, rats first learn a set of constant place-flavor associations over the course of 6 weeks before being presented with a new association followed by hippocampal lesions afterwards. The findings demonstrate markedly accelerated systems consolidation gradient as recall of the new association was only impaired if rats received lesions within $3 \mathrm{~h}$ after learning (Tse et al, 
a
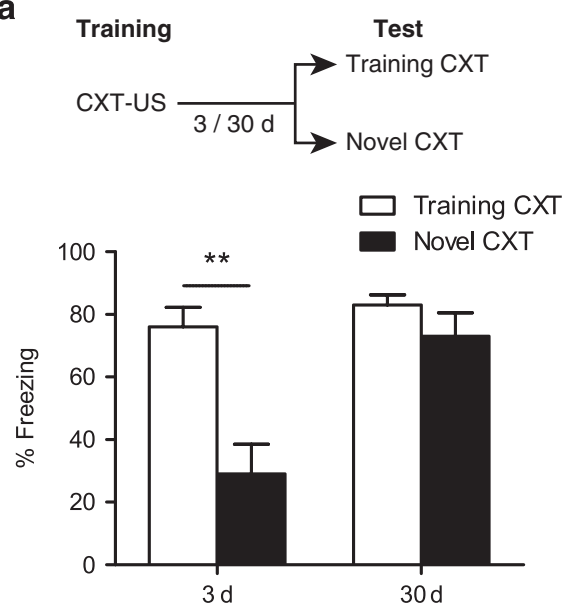

C
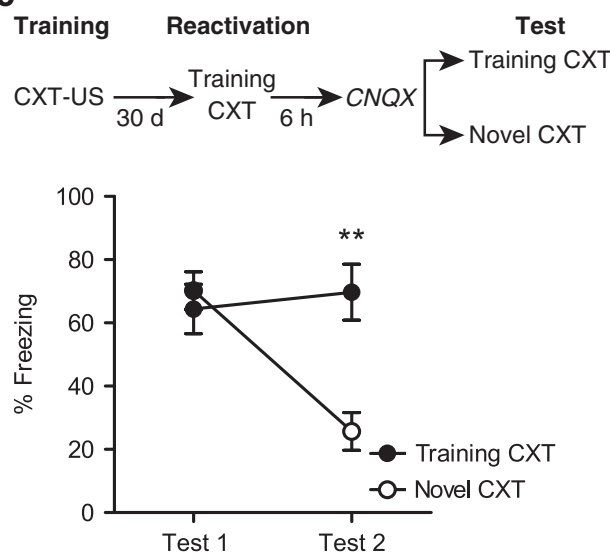

b
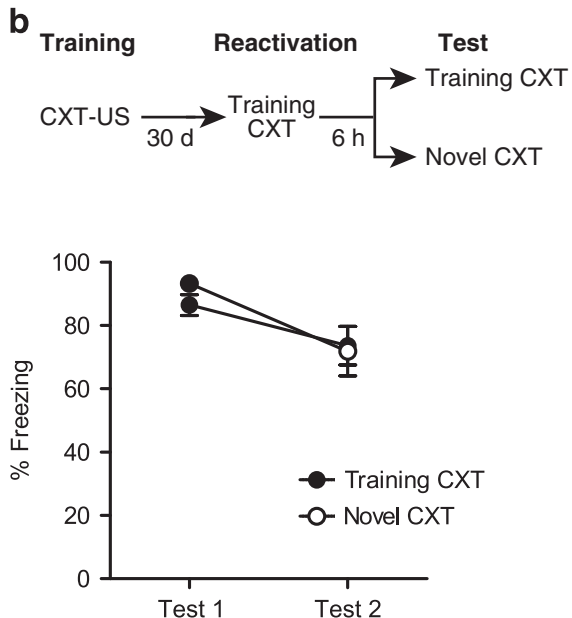

d
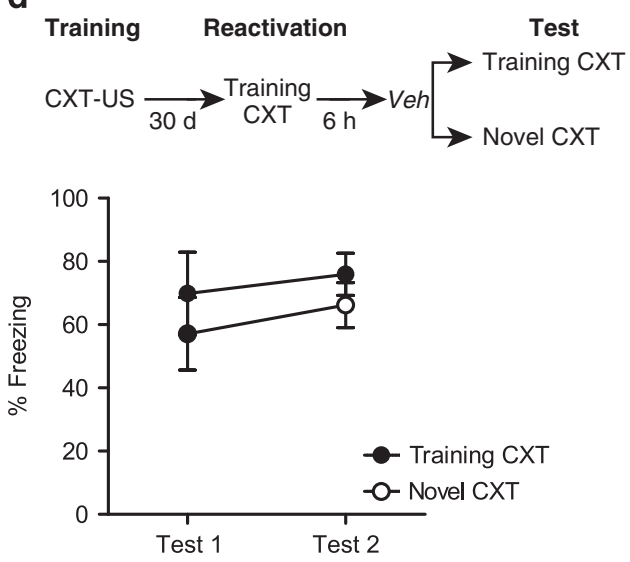

Figure 3 Effects of CNQX infusion into the ACC on generalized contextual fear memory preceded by memory reactivation. (a, upper) Experimental design used with data presented below. (a, lower) When tested 3 days after training, rats showed high levels of freezing in the original training context, but not in a novel context. At 30 days, however, rats showed contextual fear generalization with similar levels of freezing to both the original training context and a novel context. (b, upper) Experimental design used with data presented below. (b, lower) Remote memory reactivation in the original training context (test I) did not affect contextual generalization $6 \mathrm{~h}$ later (test 2) where similar high levels of freezing were expressed in both the training context and the novel context. (c, upper) Experimental design used with data presented below. (c, lower) At $6 \mathrm{~h}$ following the reactivation of a 30-day-old memory, CNQX infusions into the ACC impaired contextual fear expression in the novel context, whereas vehicle infusions into the ACC did not impair contextual fear expression in either the training or the novel context (d). ***P<0.0I. Data are means \pm SEM.

2007), after which the memory became dependent on the ACC (Wang et al, 2012).

In contrast to our finding that memory expression can be supported by the dorsal hippocampus during ACC inactivation $6 \mathrm{~h}$ after remote memory reactivation, but not after $24 \mathrm{~h}$, previous studies (Debiec et al, 2002; Winocur et al, 2009) found that electrolytic lesions of the dorsal hippocampus applied up to $24 \mathrm{~h}$ after reactivation effectively disrupted memory expression 7 days later. Although these findings might seem contradictory at first, there are notable differences between the experiments that might account for the different results, such as different methods of manipulation (transient pharmacological inactivation vs permanent electrolytic lesioning), and different delays between manipulation and test (immediate vs 7 days). Acute local pharmacological suppression of neural activity in a brain structure is, first, more specific as fibers of passage are unaffected, and second, allows for immediate behavioral testing after drug administration. In contrast, electrolytic lesions of a brain structure affect fibers of passage, and typically require a recovery period of a week before behavioral testing (Debiec et al, 2002; Winocur et al, 2009).

A number of studies have described how contextual fear memory becomes less context-specific over time, showing that animals increasingly generalize conditioned responding to novel contexts (Biedenkapp and Rudy, 2007; Ruediger et al, 2011; Wiltgen and Silva, 2007; Wiltgen et al, 2010; Winocur et al, 2009; Winocur et al, 2007). Similarly, we found that rats did not generalize conditioned fear responding to a novel context when tested 3 days after training, but expressed generalized fear in the novel context when tested 30 days after training. Moreover, our finding that AMPA/kainate-receptor blockade in the ACC $6 \mathrm{~h}$ after memory reactivation specifically disrupts fear expression in the novel context suggests that the ACC has a selective role in the expression of generalized contextual fear memory. This finding is consistent with the transformation hypothesis according to which the hippocampus supports detailed, context-specific memories, which are over time transformed into gist-like, context-unspecific representations supported 
by cortical structures (Winocur et al, 2010). This posited role of the hippocampus in supporting context memory precision is supported by recent studies showing (a) that inactivation of the dorsal hippocampus disrupts the expression of context-specific fear memory, but not the expression of generalized fear memory (de Oliveira Alvares et al, 2012; Wiltgen et al, 2010) and (b) that inhibitory connectivity in the mossy fibers of the dorsal hippocampus are critical for maintaining context-specificity of contextual fear memory (Ruediger et al, 2011).

Previous studies have demonstrated that brief re-exposure to a training context at remote time points can reinstate context-specific memory expression, an effect that lasts from $1 \mathrm{~h}$ (Zhou and Riccio, 1994), up to at least $24 \mathrm{~h}$ (Wiltgen and Silva, 2007). This brief reversal of fear generalization may be mediated by a transient recruitment of the hippocampus, possibly as part of a memory updating process. Although in our study remote memory reactivation did not lead to renewed context-specificity of memory expression $6 \mathrm{~h}$ later, memory reactivation led fear expression in the training context to being insensitive to suppression of neural activity in either the ACC or the dorsal hippocampus, but not both, whereas expression in the novel context remained ACC dependent. This finding suggests that a highly dynamic transient process involving the hippocampus and the ACC mediates the expression of these remote memories after reactivation, which is not fully accounted for by the transformation hypothesis. One possibility is that this reinstatement of dorsal-hippocampus supported fear expression in the training context, during suppression of neural activity in the ACC, reflects a residual context-specific hippocampal memory trace that has decayed over time but has been re-strengthened by memory reactivation (Hardt et al, 2013), although not to the point of overriding a stronger ACC-dependent context-generalized fear memory. Another possibility is that the hippocampussupported fear expression reflects a new memory of the training context from the memory reactivation session $6 \mathrm{~h}$ earlier, and which could be part of a memory updating process of the hippocampal-cortical memory trace. Such a process would be consistent with the idea that retrieval can initiate re-storage of the memory in a new hippocampal trace (Nadel and Moscovitch, 1997).

Moreover, although we found that contextual fear memory expression returned to being ACC-dependent $24 \mathrm{~h}$ after memory reactivation when tested in the training context, it remains to be examined whether fear expression returns to being context-specific at this time point and whether the hippocampus remains involved in supporting such fear expression. A finding of ACC-dependent context-specific fear expression would neither be consistent with the transformation hypothesis nor the standard model of systems consolidation (Squire and Alvarez, 1995), which does not predict or account for how systems consolidated memories change not only their quality but also their anatomical loci of dependence following memory reactivation.

An alternative account of the role of the ACC in supporting remote generalized memory expression proposes that memory degrades over time, which makes it harder to retrieve, and that the ACC facilitates the retrieval of degraded memories (Biedenkapp and Rudy, 2007; Rudy et al, 2005). According to this view, recent context-specific memory is an example of a memory that does not require the ACC to be expressed, whereas a remote generalized memory is an example of a weakened memory trace that cannot be expressed without the ACC. In contrast, we found that the ACC specifically mediates the expression of generalized fear memory and not context-specific memory, as suppressing neural activity in the ACC $6 \mathrm{~h}$ after memory reactivation impaired contextual fear expression in a novel context, but not in the training context.

The nature of the interaction between the ACC and the hippocampus in memory consolidation is currently the subject of intense debate. A number of studies have demonstrated that hippocampal lesions or pharmacological inactivation disrupt recent contextual fear memories more than remote ones (Anagnostaras et al, 1999; de Oliveira Alvares et al, 2012; Goshen et al, 2011; Kitamura et al, 2009; Ward et al, 1999; Wiltgen et al, 2010; Winocur et al, 2009). Furthermore, suppressing neural activity in the ACC impairs expression of remote contextual fear memories more than the expression of recent ones (Frankland et al, 2004; Goshen et al, 2011). However, other studies revealed a different pattern of findings. Local infusions of a protein synthesis inhibitor in the ACC (Einarsson and Nader, 2012), or the dorsal hippocampus (Debiec et al, 2002), following recent or remote memory reactivation disrupt contextual fear memory, and optogenetic inhibition of CA1 of the dorsal hippocampus disrupts expression of both recent and remote contextual fear (Goshen et al, 2011). Moreover, selective inactivation of medial prefrontal neurons (including the ACC, prelimbic, and infralimbic cortices) projecting to the nucleus reuniens during contextual fear conditioning leads to enhanced generalization on a later test $\mathrm{Xu}$ and Sudhof, 2013). This finding suggests that specific circuits in the medial prefrontal cortex may be involved in encoding memory specificity, although it is not clear if the circuit overlaps with the one affected in our study. Together with these findings, our results suggest that the ACC and dorsal hippocampus are dynamically involved in supporting both recent and remote contextual fear memory, with the hippocampus having a more significant role in the expression of context-specific fear memory, and the ACC having a more significant role in the expression of generalized fear memory. Furthermore, our findings suggest that memory reactivation leads to renewed involvement of the dorsal hippocampus, which, together with the ACC, might be involved in the incorporation of new information into an existing memory trace. Such a putative process would fit well with findings in humans suggesting that retrieval may modify the retrieved memory (Bartlett, 1932; Hardt et al, 2010).

\section{FUNDING AND DISCLOSURE}

The authors declare no conflict of interest.

\section{ACKNOWLEDGEMENTS}

We thank Oliver Hardt and Cyril Herry for helpful comments on the manuscript. This work was supported by grants from the Canadian Institutes of Health Research and the Natural Sciences and Engineering Research Council of Canada (to KN). 


\section{REFERENCES}

Anagnostaras SG, Maren S, Fanselow MS (1999). Temporally graded retrograde amnesia of contextual fear after hippocampal damage in rats: within-subjects examination. J Neurosci 19: $1106-1114$.

Attwell PJ, Rahman S, Ivarsson M, Yeo CH (1999). Cerebellar cortical AMPA-kainate receptor blockade prevents performance of classically conditioned nictitating membrane responses. J Neurosci 19: RC45.

Bartlett FC (1932). Remembering: A Study in Experimental and Social Psychology. Cambridge University Press: Cambridge.

Biedenkapp JC, Rudy JW (2007). Context preexposure prevents forgetting of a contextual fear memory: implication for regional changes in brain activation patterns associated with recent and remote memory tests. Learn Mem 14: 200-203.

Corcoran KA, Desmond TJ, Frey KA, Maren S (2005). Hippocampal inactivation disrupts the acquisition and contextual encoding of fear extinction. J Neurosci 25: 8978-8987.

de Oliveira Alvares L, Einarsson EO, Santana F, Crestani AP, Haubrich J, Cassini LF et al (2012). Periodically reactivated context memory retains its precision and dependence on the hippocampus. Hippocampus 22: 1092-1095.

Debiec J, LeDoux JE, Nader K (2002). Cellular and systems reconsolidation in the hippocampus. Neuron 36: 527-538.

Einarsson EO, Nader K (2012). Involvement of the anterior cingulate cortex in formation, consolidation, and reconsolidation of recent and remote contextual fear memory. Learn Mem 19: 449-452.

Flavell CR, Barber DJ, Lee JL (2011). Behavioural memory reconsolidation of food and fear memories. Nat Commun 2: 504.

Frankland PW, Bontempi B (2005). The organization of recent and remote memories. Nat Rev Neurosci 6: 119-130.

Frankland PW, Bontempi B, Talton LE, Kaczmarek L, Silva AJ (2004). The involvement of the anterior cingulate cortex in remote contextual fear memory. Science 304: 881-883.

Goshen I, Brodsky M, Prakash R, Wallace J, Gradinaru V, Ramakrishnan C et al (2011). Dynamics of retrieval strategies for remote memories. Cell 147: 678-689.

Hardt O, Einarsson EO, Nader K (2010). A bridge over troubled water: reconsolidation as a link between cognitive and neuroscientific memory research traditions. Annu Rev Psychol 61: 141-167.

Hardt O, Nader K, Nadel L (2013). Decay happens: the role of active forgetting in memory. Trends Cogn Sci 17: 111-120.

Honore T, Davies SN, Drejer J, Fletcher EJ, Jacobsen P, Lodge D et al (1988). Quinoxalinediones: potent competitive non-NMDA glutamate receptor antagonists. Science 241: 701-703.

Kitamura T, Saitoh Y, Takashima N, Murayama A, Niibori Y, Ageta $\mathrm{H}$ et al (2009). Adult neurogenesis modulates the hippocampusdependent period of associative fear memory. Cell 139: 814-827.

McClelland JL, McNaughton BL, O'Reilly RC (1995). Why there are complementary learning systems in the hippocampus and neocortex: insights from the successes and failures of connectionist models of learning and memory. Psychol Rev 102: 419-457.
Monfils MH, Cowansage KK, Klann E, LeDoux JE (2009). Extinction-reconsolidation boundaries: key to persistent attenuation of fear memories. Science 324: 951-955.

Nadel L, Moscovitch M (1997). Memory consolidation, retrograde amnesia and the hippocampal complex. Curr Opin Neurobiol 7: 217-227.

Nader K, Schafe GE, Le Doux JE (2000). Fear memories require protein synthesis in the amygdala for reconsolidation after retrieval. Nature 406: 722-726.

Riccio DC, Ackil J, Burch-Vernon A (1992). Forgetting of stimulus attributes: methodological implications for assessing associative phenomena. Psychol Bull 112: 433-445.

Rudy JW, Biedenkapp JC, O'Reilly RC (2005). Prefrontal cortex and the organization of recent and remote memories: an alternative view. Learn Mem 12: 445-446.

Ruediger S, Vittori C, Bednarek E, Genoud C, Strata P, Sacchetti B et al (2011). Learning-related feedforward inhibitory connectivity growth required for memory precision. Nature 473: 514-518.

Squire LR, Alvarez P (1995). Retrograde amnesia and memory consolidation: a neurobiological perspective. Curr Opin Neurobiol 5: 169-177.

Tse D, Langston RF, Kakeyama M, Bethus I, Spooner PA, Wood ER et al (2007). Schemas and memory consolidation. Science 316: 76-82.

Vetere G, Restivo L, Cole CJ, Ross PJ, Ammassari-Teule M, Josselyn SA et al (2011). Spine growth in the anterior cingulate cortex is necessary for the consolidation of contextual fear memory. Proc Natl Acad Sci USA 108: 8456-8460.

Wang SH, Tse D, Morris RG (2012). Anterior cingulate cortex in schema assimilation and expression. Learn Mem 19: 315-318.

Ward MT, Oler JA, Markus EJ (1999). Hippocampal dysfunction during aging I: deficits in memory consolidation. Neurobiol Aging 20: 363-372.

Wiltgen BJ, Silva AJ (2007). Memory for context becomes less specific with time. Learn Mem 14: 313-317.

Wiltgen BJ, Zhou M, Cai Y, Balaji J, Karlsson MG, Parivash SN et al (2010). The hippocampus plays a selective role in the retrieval of detailed contextual memories. Curr Biol 20: 1336-1344.

Winocur G, Frankland PW, Sekeres M, Fogel S, Moscovitch M (2009). Changes in context-specificity during memory reconsolidation: selective effects of hippocampal lesions. Learn Mem 16: 722-729.

Winocur G, Moscovitch M, Bontempi B (2010). Memory formation and long-term retention in humans and animals: convergence towards a transformation account of hippocampal-neocortical interactions. Neuropsychologia 48: 2339-2356.

Winocur G, Moscovitch M, Sekeres M (2007). Memory consolidation or transformation: context manipulation and hippocampal representations of memory. Nat Neurosci 10: 555-557.

Xu W, Sudhof TC (2013). A neural circuit for memory specificity and generalization. Science 339: 1290-1295.

Zhou YL, Riccio DC (1994). Pretest cuing can alleviate the forgetting of contextual stimulus attributes. Learn Motiv 25: 233-244.

Supplementary Information accompanies the paper on the Neuropsychopharmacology website (http://www.nature.com/npp) 